\title{
Progression of breast tumors is accompanied by a decrease in expression of the Rho guanine exchange factor Tiam1
}

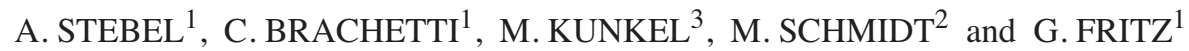 \\ ${ }^{1}$ Department of Toxicology, University of Mainz; Departments of ${ }^{2}$ Obstetrics and Gynecology, \\ ${ }^{3}$ Oral and Maxillofacial Surgery, University Hospital Mainz, Germany
}

Received June 5, 2008; Accepted July 22, 2008

DOI: $10.3892 /$ or_00000211

\begin{abstract}
In this study, we investigated the expression level of Ras-homologous (Rho) GTPases and the Rho guanine exchange factor (GEF) T-cell lymphoma invasion and metastasis 1 (Tiam1) in breast tumor specimens $(n=106)$ by immunohistochemistry. Rho and Rho-GEF expression scores were compared to clinically established diagnostic and prognostic parameters. We found that RhoA and RhoB scores slightly increased with tumor grade, whereas the Rac1 score remained unaffected. The most significant effects were observed for the Rac1-specific GEF Tiam1. Tiam1 expression scores significantly decreased with the increase in tumor grade, tumor spreading and proliferation. Furthermore, Tiam1 expression was inversely related to the plasminogen activator inhibitor (PAI-1) and estrogen receptor (ER) expression but not the progesterone receptor (PR) and urokinase plasminogen activator (uPA). A low Tiam1 expression was associated with p53 positivity without being related to HER2/neu status. The data show that Tiam1 expression decreases with the progression of breast carcinomas and is inversely associated with several established breast tumor markers. Therefore, we suggest that Tiam1 counteracts the progression of breast carcinomas and is suitable as a novel breast tumor marker.
\end{abstract}

\section{Introduction}

Tumor progression and metastasis are characterized by a gain in proliferation, migration and invasive properties of tumor cells. Additionally, programmed cell death, which normally occurs as a consequence of loss of cell adhesion, needs to be

Correspondence to: Dr Gerhard Fritz, University of Mainz, Department of Toxicology, Obere Zahlbacher Str. 67, D-55131 Mainz, Germany

E-mail: fritz@uni-mainz.de

Abbreviations: Rho, Ras-homologous; ER, estrogen receptor; GEF, guanine exchange factor; PR, progesterone receptor; Tiam, T-cell lymphoma invasion and metastasis 1

Key words: Ras homologous GTPases, Rho guanine exchange factors, breast tumor markers, oncogenes, immunohistochemistry by-passed. These alterations require fundamental changes in the cellular gene expression programme. Low molecular weight GTPases of the Rho family are central players in the regulation of many of these processes as demonstrated by numerous in vitro and in vivo studies (1-6). Western blot and RNA-based data show that Rho expression is related to the aggressiveness of several solid tumor types, including pancreas (7) and breast carcinomas (8-11). Surprisingly, an inverse association between Rho expression and the malignancy of brain tumors has been reported (12). Furthermore, breast and colon cancer revealed an enhanced expression of the Rac splice variant Rac 1b (13). Overexpression of Rho GTPases in tumors is suggested as a factor contributing to the putative anticancer effects of statins $(14,15)$. Moreover, RhoB (16) and RhoE (17) were reported to have a tumor-suppressing function. The putative tumor suppressor property of RhoB may be related to its proapoptotic activity $(18,19)$.

Apart from tumor-specific changes in the expression level of Rho proteins, it is assumed that the activity (i.e. GTPbinding) status of Rho proteins undergoes time- and spatialdependent fluctuations during the different steps involved in tumor progression and metastasis $(2,20)$. As the activity status of Rho GTPases is regulated by Rho regulatory factors including guanine exchange factors (GEFs) such as Dbl, Tiam1 and Vav1, which are known to be oncogenic (21), changes in the expression level of these factors may also change with malignancy. For instance, the expression of Tiam1 (T-cell lymphoma invasion and metastasis 1) was found to be up-regulated in a subset of colorectal carcinomas (22). Apart from Rho GEFs, the expression level of GTPaseactivating proteins (GAPs) or guanine dissociation-inhibiting proteins (Rho-GDI) may vary with malignancy, as a low RhoGDI expression was recently observed in highly malignant grade III glioblastoma (23). Thus, tumor-specific changes in Rho-regulated signaling may be reflected on the level of Rho protein expression and the expression level of Rho-regulatory factors.

Semiquantitative expression analysis of Rho GTPases and Rho-regulatory factors in malignant tissue by means of immunohistochemistry is lacking. However, this analysis is important as it supports the concept that they are of functional relevance for tumor progression, establishing them as clinically useful diagnostic and/or prognostic markers. Therefore, in the present study we analyzed the expression 
level of different types of Rho GTPases and GEFs in breast tumors, by employing immunohistochemistry. The obtained Rho and Rho GEF expression scores were related to clinically relevant breast tumor markers including tumor grade, spreading and type, and proliferation as well as the expression status of uPA, PAI-1, p53, Her2/neu and hormone receptors. The results obtained show that the expression score of the Rac1-specific GEF Tiam1 is significantly and inversely associated with several established breast tumor markers. This finding points to a particular relevance of Tiam1 for breast tumor progression and indicates that Tiam1 is useful as a novel breast tumor marker.

\section{Materials and methods}

Materials. For morphological and immunohistochemical evaluation, routinely processed paraffin blocks of formalinfixed tumorigenic tissues from breast specimens were used. The histological grade of malignancy (G1/G2/G3), Ki-67 proliferation index (MIB-1), tumor spreading (T1-T4), tumor type (ductal, lobular and mixed), lymph node status, invasiveness, expression of urokinase plasminogen activator (uPA) and uPA-inhibitor type 1 (PAI-1), as well as p53, HER-2/neu oncogene and hormone receptor status (ER and PR) were established within routine pathological assessment. The $n=105$ paraffin-embedded breast tumor specimens (which underwent pathological consideration) were included in the study. RhoA(no. sc-179, rabbit polyclonal), RhoB- (no. sc-180, rabbit polyclonal) as well as Tiam1- (no. sc-872, rabbit polyclonal) and Dbl- (no. sc-28582, rabbit polyclonal) specific antibodies were purchased from Santa Cruz (San Diego, USA). Rac1 (no. R56220, mouse monoclonal), Cdc42 (no. C70820, mouse monoclonal) and Vav1 (no. 07192) antibodies were purchased from Upstate (Hamburg, Germany).

Immunohistochemistry. Expression analysis of Rho and Rho regulatory factors in breast tissue was performed by conventional immunohistochemistry. Paraffin-embedded blocks of the formalin-fixed tumor samples were retrieved from the pathology archives of the Department of Obstetrics and Gynecology (University Hospital Mainz, Germany). Sections were immunostained for Rho GTPases (i.e. RhoA, RhoB, Rac1 and Cdc42) and Rho GEFs (Tiam1, Vav1 and Dbl) using the avidin biotin technique according to the standard protocol. Briefly, $6 \mu \mathrm{m}$ sections were deparaffinized and rehydrated. Antigen retrieval by exposure of the section to hot steam [20 min in $1 \mathrm{mM}$ EDTA buffer (pH 8.0)] was necessary only when Rac1-, Vav1- and Dbl-specific antibodies were used. Sections were quenched for endogenous peroxidase activity with $0.1 \% \mathrm{H}_{2} \mathrm{O}_{2}$ treatment $(20 \mathrm{~min})$ and then incubated with $10 \%$ normal goat serum $(30 \mathrm{~min})$ to block the non-specific binding of immunoglobulins to the tissue, followed by incubation with the corresponding antibodies (at a final dilution of 1:100-1:250; incubation overnight at room temperature). The ChemMate ${ }^{\mathrm{TM}}$ Dako Envision detection kit (Dako Diagnostics, Hamburg, Germany) was used for visualization. Nuclei were lightly counterstained with hematoxylin. A microscopical calculation of the Rho/Rho-GEF scoring index in a given tumor sample was calculated by determining (i) the percentage of tumor cells that expressed the protein
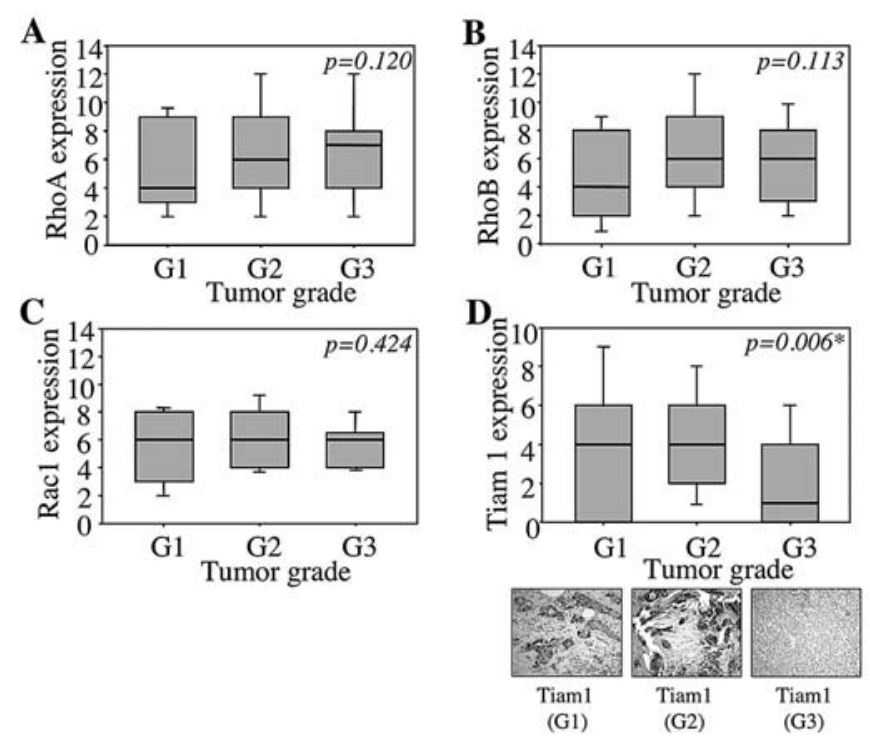

Figure 1. Association between the expression of Rho GTPases, Tiam1 and tumor grade. (A) RhoA expression scores in G1 $(n=27), G 2(n=38)$ and G3 $(n=40)$ grade tumors. Data are shown in a box-blot. The horizontal line shows the median. The end of the boxes represents the 25 th and 75 th percentiles. The error bars define the 10th and 90th percentiles. Statistical analysis was performed using the Kruskal-Wallis test. ${ }^{*} \mathrm{p} \leq 0.05$ was considered as a statistically significant difference. (B) RhoB and (C) Rac1 expression scores in G1, G2 and G3 grade tumors. Presentation of the data and statistical analysis were performed as described in (A). (D) Tiam1 expression scores in G1 $(n=27), G 2(n=38)$ and G3 $(n=36)$ grade tumors. Presentation of the data and statistical analysis were performed as described in (A). The bottom right side of the figure shows the expression of Tiam1 in representative samples of G1-G3 grade tumors.

[score $0(<10 \%$ of stained tumor cells) to 4 ( $>80 \%$ tumor cells stained)] and (ii) staining intensity [score 0 (no staining) to 3 (high staining)] resulting in an overall score ranging from 0 to 12 . The data obtained are presented in a box-blot. Differences between two independent groups were analyzed for statistical significance using the Mann-Whitney U test, while differences among the groups were analyzed by the Kruskal-Wallis test. To check for correlation between the two factors, Spearman's test was applied. Two-sided P-values $<0.05$ were considered as statistically significant. Statistical analysis was performed using SigmaStat 3.5 software.

\section{Results and Discussion}

In the present study, we investigated the expression of Rho GTPases and GEFs in a breast tumor specimen by immunohistochemistry. We found that the expression level of the Rho GTPases RhoA, RhoB, Rac1 and Cdc42 as well as that of Rho GEFs Tiam1, Vav1 and Dbl is specifically enhanced in the malignant cells of breast as compared to the surrounding stroma cells (data not shown). In order to determine whether the immunohistochemically detectable expression of Rho and Rho GEFs is related to clinically established and relevant tumor markers of the breast, we investigated the expression of RhoA, RhoB, Rac1 and Tiam1 in $n=105$ breast tumor samples. The protein expression of Rho GTPases and Tiam1 was scored by combining the staining intensity and the percentage of tumor cells expressing Rho or Tiam1 protein. 
Table I. Rho and Tiam1 expression is related to the grade of malignancy (G1/G2/G3) of breast tumors.

\begin{tabular}{lccc}
\hline Parameter & $\begin{array}{c}\text { Staining intensity } \\
(\text { score } 1-3)\end{array}$ & $\begin{array}{c}\text { \% Positive cells } \\
\text { (score 1-4) }\end{array}$ & $\begin{array}{c}\text { Expression score }^{\mathrm{b}} \\
(\text { score } 0-12)\end{array}$ \\
\hline RhoA & $\mathrm{p}=0.345$ & $\mathrm{p}=0.006^{\mathrm{a}}$ & $\mathrm{p}=0.120$ \\
RhoB & $\mathrm{p}=0.124$ & $\mathrm{p}=0.067$ & $\mathrm{p}=0.113$ \\
Rac1 & $\mathrm{p}=0.606$ & $\mathrm{p}=0.055$ & $\mathrm{p}=0.424$ \\
Tiam1 & $\mathrm{p}=0.093$ & $\mathrm{p} \leq 0.001^{\mathrm{a}}$ & $\mathrm{p}=0.006^{\mathrm{a}}$ \\
\hline
\end{tabular}

${ }^{a}$ Statistical significance (based on Kruskal-Wallis test). ${ }^{\mathrm{b}}$ Calculated from staining intensity and percentage of positive cells.

A

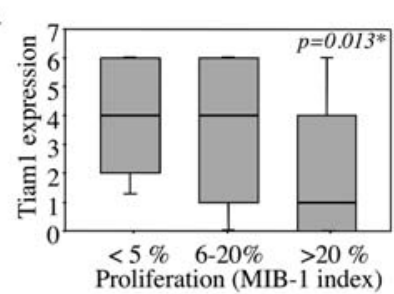

B

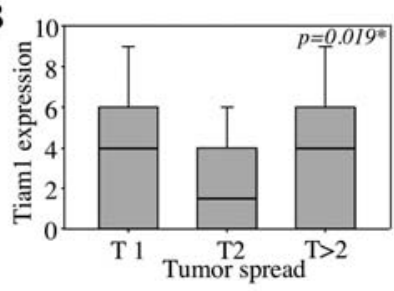

C

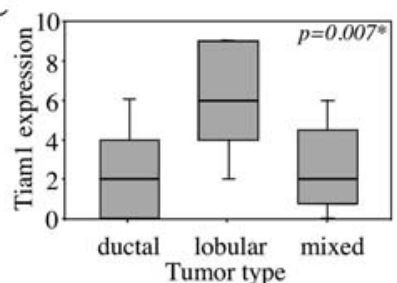

D

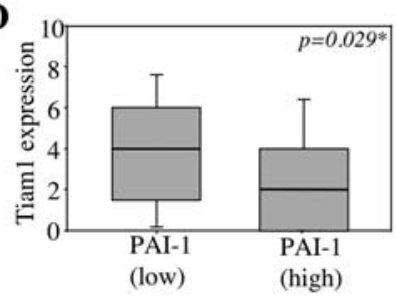

Figure 2. Tiam1 expression score and its relation to proliferation, tumor spreading, tumor type and expression of PAI-1. The expression score of Tiam1 was related to the (A) proliferation state of the tumor (MIB-1), (B) tumor spread (T1, T2 and >T2), (C) tumor type and (D) expression level of the urokinase plasminogen activator inhibitor type 1 (PAI-1). PAI-1 (low), amount of PAI- 1 is $<13 \mathrm{ng} / \mathrm{mg}$ protein and PAI-1 (high), amount of PAI-1 is $>13 \mathrm{ng} / \mathrm{mg}$ protein. ${ }^{*} \mathrm{p} \leq 0.05$ was considered as a statistically significant difference.

There was no statistically significant difference detectable between the expression scores of RhoA (Fig. 1A), RhoB (Fig. 1B) and Rac1 (Fig. 1C) and the grade of malignancy. Only the percentage of RhoA-expressing cells significantly increased with the tumor grade (Table I). A similar tendency, although statistically not significant, was also seen for RhoB and Rac1 (Table I). A pairwise statistical analysis revealed a significant increase in RhoB expression from G1 to G2 grade tumors. G1 to G2 progression tends to result in an increase in the RhoA score (Table II). Since Rac1 expression did not change with histological grading (Fig. 1C, Tables I and II), the effects observed for RhoA and RhoB are considered as specific. Notably, the Tiam1 expression score significantly declined with the grade of malignancy (Fig. 1D), in particular with the progression from G2 to G3 grade tumors (Table II). As Tiam1 is a Rac1-specific guanine nucleotide exchange factor $(24,25)$, the outcome of a reduced expression of Tiam 1 can be assumed to be a decline in Rac1 activity. Consequently, a reduction in Rac1 activity may collaborate with the ongoing malignancy of breast tumors. Interestingly, RhoA and Rac1 have been reported to exert opposing biological effects

Table II. Rho and Tiam1 expression scores in breast tumors of different grading.

\begin{tabular}{llll}
\hline Parameter & $\mathrm{G} 1 / \mathrm{G} 2$ & $\mathrm{G} 2 / \mathrm{G} 3$ & $\mathrm{G} 1 / \mathrm{G} 3$ \\
\hline RhoA & $\mathrm{p}=0.055$ & $\mathrm{p}=0.784$ & $\mathrm{p}=0.088$ \\
RhoB & $\mathrm{p}=0.044^{\mathrm{a}}$ & $\mathrm{p}=0.649$ & $\mathrm{p}=0.116$ \\
Rac1 & $\mathrm{p}=0.377$ & $\mathrm{p}=0.199$ & $\mathrm{p}=0.920$ \\
Tiam1 & $\mathrm{p}=0.466$ & $\mathrm{p} \leq 0.001^{\mathrm{a}}$ & $\mathrm{p}=0.052$ \\
\hline
\end{tabular}

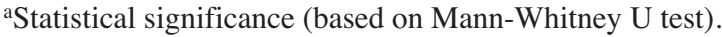

$(9,25,26)$. With these reports in mind, we speculate that the reduced Tiam1 expression, and concomitant decrease in Rac1 activity, eventually promotes RhoA-regulated functions which are required for the progression of breast tumors. RhoC has also been suggested as a biomarker for aggressive breast carcinomas because a high $\mathrm{RhoC}$ expression was found to be predictive of poor survival of breast cancer patients (11). Thus, RhoA and RhoC can functionally compensate for each other (9).

The presence of lymph node metastases was neither significantly associated with the expression of Rho GTPases nor with Tiam1 (data not shown). However, a high proliferation index (MIB-1) was significantly related to a reduction in the Tiam1 score (Fig. 2A). An increase in tumor spread (Tclassification) was accompanied by a decrease of the Tiam1 expression score (Fig. 2B). Interestingly, lobular breast tumors revealed a significantly higher Tiam1 expression than ductal or mixed-type tumors (Fig. 2C). A significant association of Tiam1 expression was also observed with respect to plasminogen activator inhibitor type 1 (Fig. 2D), with a high PAI-1 expression ( $>13 \mathrm{ng} / \mathrm{ml}$ protein) being related to a low Tiam1 score. An association of Tiam1 score with the expression level of urokinase plasminogen activator (uPA) was not observed (Table III). No statistically significant correlations were detected between the expression scores of Rho GTPases and MIB-1, tumor size, tumor type, uPA or PAI-1 (Table III).

We then investigated whether Rho and Tiam1 expression are associated with the p53, Her2/neu or hormone receptor status of breast tumors. We found p53 positivity to be significantly related to a high RhoA and a low Tiam1 score (Fig. 3A and B). RhoB and Rac1 expression were not 
Table III. Summary of the associations observed between Rho and Tiam1 expression scores and clinically established diagnostic and prognostic parameters of breast tumors.

\begin{tabular}{|c|c|c|c|c|}
\hline Parameter & RhoA score & RhoB score & Rac1 score & Tiam1 score \\
\hline $\begin{array}{l}\text { Tumor grade } \\
(\mathrm{G} 1 / \mathrm{G} 2 / \mathrm{G} 2)\end{array}$ & NS & NS & NS & $\mathrm{p} \leq 0.01$ \\
\hline Grade $1 / 2^{c}$ & $\mathrm{NS}^{\mathrm{a}}$ & $\mathrm{p} \leq 0.05$ & NS & NS \\
\hline Grade $2 / 3^{c}$ & NS & NS & NS & $\mathrm{p} \leq 0.001$ \\
\hline $\begin{array}{l}\text { Lymph node status }^{c} \\
\text { (positive/negative) }\end{array}$ & NS & NS & NS & NS \\
\hline $\begin{array}{l}\text { Proliferation index } \\
(<5 /<20 />20 \%)\end{array}$ & NS & NS & NS & $\mathrm{p} \leq 0.02$ \\
\hline $\begin{array}{l}\text { Tumor spreading }{ }^{\mathrm{b}} \\
(\mathrm{T} 1 / \mathrm{T} 2 />\mathrm{T} 2)\end{array}$ & NS & NS & NS & $\mathrm{p} \leq 0.02$ \\
\hline $\begin{array}{l}\text { Invasiveness }^{c} \\
\text { (positive/negative) }\end{array}$ & NS & NS & NS & NS \\
\hline $\begin{array}{l}\text { Tumor type } \\
\text { (ductal/lobular/mixed) }\end{array}$ & NS & NS & NS & $\mathrm{p} \leq 0.01$ \\
\hline $\begin{array}{l}\mathrm{uPA}^{\mathrm{c}} \\
\qquad(<3 />3 \mathrm{ng} / \mathrm{ml})\end{array}$ & NS & NS & NS & NS \\
\hline $\begin{array}{l}\text { PAI- }{ }^{\mathrm{c}} \\
\qquad(<13 />13 \mathrm{ng} / \mathrm{ml})\end{array}$ & NS & NS & NS & $\mathrm{p} \leq 0.03$ \\
\hline $\begin{array}{l}\text { p53 status }^{\mathrm{c}} \\
\text { (positive/negative) }\end{array}$ & $\mathrm{p} \leq 0.03$ & $\mathrm{NS}^{\mathrm{a}}$ & NS & $\mathrm{p} \leq 0.05$ \\
\hline $\begin{array}{l}\text { HER2/neu status }^{c} \\
\text { (positive/negative) }\end{array}$ & $\mathrm{NS}^{\mathrm{a}}$ & NS & NS & NS \\
\hline ER score ${ }^{d}$ & NS & $\mathrm{NS}^{\mathrm{a}}$ & NS & $\mathrm{NS}^{\mathrm{a}}$ \\
\hline $\begin{array}{l}\text { ER status }^{c} \\
\text { (low/high) }\end{array}$ & $\mathrm{NS}^{\mathrm{a}}$ & NS & NS & $\mathrm{p} \leq 0.01$ \\
\hline PR score ${ }^{d}$ & NS & NS & NS & $\mathrm{NS}^{\mathrm{a}}$ \\
\hline $\begin{array}{l}\text { PR status }{ }^{c} \\
\text { (low/high) }\end{array}$ & NS & NS & NS & NS \\
\hline
\end{tabular}

NS, not significant ( $p>0.05)$; NS ${ }^{a}$, not significant, but with a tendency towards significance $(\mathrm{p} \leq 0.1)$; ${ }^{b}$ statistical analysis according to the Kruskal-Wallis test; ${ }^{\mathrm{c}}$ statistical analysis according to the Mann-Whitney U test; ${ }^{\mathrm{d}}$ statistical analysis according to Spearman's correlation test.

A

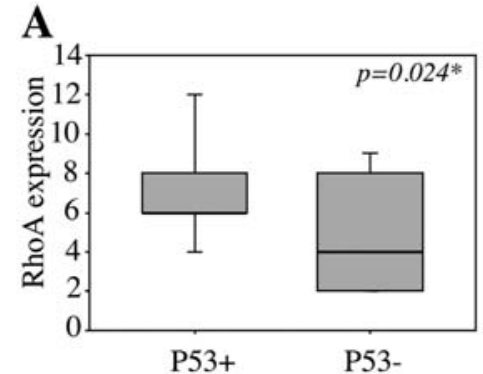

B

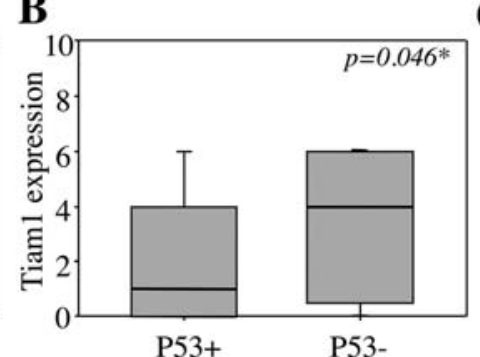

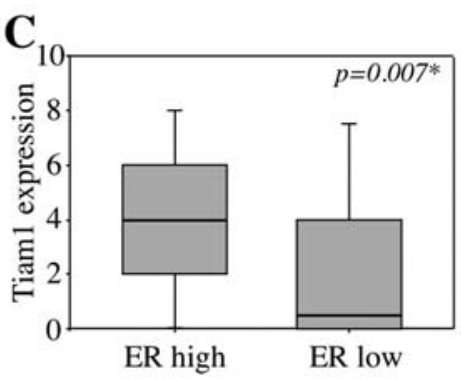

Figure 3. Expression levels of RhoA and Tiam1 are associated with p53 expression and estrogen receptor status of breast tumors. (A and B) The expression scores of (A) RhoA and (B) Tiam1 were related to p53 expression. p53+ and p53-, tumors either stained (p53+) or not (p53-) by p53-specific antibody. ${ }^{*} \mathrm{p} \leq 0.05$ was considered as significant. (C) Expression score of Tiam1 is related to the expression status of the estrogen receptor (ER). Tumors with ER scores $\leq 1$ were classified as having a low ER status (ER low). Tumors with ER scores $\geq 2$ were classified as having a high ER status (ER high). ${ }^{*} \mathrm{p} \leq 0.05$ was considered as statistically significant. 
significantly related to p53 status (Table III). It should be mentioned that the monoclonal p53 antibody used (Cat\#OP43, Oncogene Research Products) detects wild-type and mutated p53. However, as the half-life of wild-type p53 is very short, immunohistochemical positivity reflects a mutated p53 protein. Regarding Her2/neu oncogene status, a clear tendency was detected for RhoA ( $\mathrm{p}=0.052)$, whereas the expression levels of the other Rho GTPases and Tiam1 were not related to the expression of this oncogene (Table III). Rho and Tiam1 scores failed to correlate with hormone receptor scores, although RhoB and Tiam1 scores tend to correlate with the ER score ( $p=0.062$ and $p=0.069$; Table III). By sub-grouping the breast tumors into tumors characterized by either a low $(\leq 1)$ or high $(>2)$ ER (or PR) score, we found that a low ER expression is associated with a low Tiam1 expression (Fig. 3C and Table III). Results of the immunohistochemical studies are summarized in Table III. It illustrates the key finding of this study, which is that the statistically most significant effects were observed for Tiam1, which is a Rac-specific GEF (25). The expression level of Tiam1 was inversely related to the grade of malignancy, tumor spreading, proliferation and PAI-1 expression as well as p53 status and is associated with a low ER expression status. Our findings are indicative of a particular relevance of Tiam1 in the progression of breast cancer. It is believed that the biological activity of Rho GTPases undergo time- and spatial-dependent fluctuations during epithelialmesenchymal transition (EMT) (20). The data obtained in this study indicate that apart from the expression level of Rho GTPases, it is in particular the expression level of the Rac1specific GEF Tiam1 that appears to contribute to a finely tuned regulation of Rho activities in the course of EMT. It is known that the biological effect of Tiam1 is cell type-specific and that Tiam 1 can promote and inhibit epithelial cell migration $(27,28)$. Regarding breast tumors, the observed decrease in Tiam1 expression collaborating with an increase in malignancy is taken as an indication that Tiam1 decelerates migration, presumably by promoting E-cadherin-mediated cell-cell contacts (28).

In summary, based on the data we suggest that the downregulation of Tiam 1 contributes to the progression of breast tumors. Therefore, Tiam1 is suitable as a novel breast tumor marker. Recently, the prognostic relevance of the Tiam1 expression was reported for prostate carcinomas (29). Since Tiam1 is required for Rac1-triggered activation of stress kinases (30), which are key players in the regulation of anticancer drug-induced apoptosis $(31,32)$, the observed changes in Tiam1 expression may impact on the therapeutic response of breast tumor cells to anticancer drugs. This important aspect will be the subject of forthcoming studies.

\section{Acknowledgements}

This work was supported by the Wilhelm Sander-Stiftung (1999.014.2 and 2005.120.1) and the Mildred Scheel Stiftung (107361). We would like to thank W.P. Roos for critical reading of the manuscript.

\section{References}

1. Fritz G and Kaina B: Rho GTPases: promising cellular targets for novel anticancer drugs. Curr Cancer Drug Targets 6: 1-14, 2006.
2. Ridley AJ: Rho proteins and cancer. Breast Cancer Res Treat 84: 13-19, 2004.

3. Ridley A: Molecular switches in metastasis. Nature 406: 466-467, 2000.

4. Sahai E and Marshall CJ: RHO-GTPases and cancer. Nat Rev Cancer 2: 133-142, 2002.

5. Jaffe AB and Hall A: Rho GTPases in transformation and metastasis. Adv Cancer Res 84: 57-80, 2002.

6. Aznar S, Fernandez-Valeron P, Espina C and Lacal JC: Rho GTPases: potential candidates for anticancer therapy. Cancer Lett 206: 181-191, 2004.

7. Suwa H, Ohshio G, Imamura T, Watanabe G, Arii S, Imamura M, Narumiya $S$, Hiai $H$ and Fukumoto M: Overexpression of the rhoC gene correlates with progression of ductal adenocarcinoma of the pancreas. Br J Cancer 77: 147-152, 1998.

8. Fritz G, Brachetti T, Bahlmann F, Schmidt M and Kaina B: Rho GTPases in human breast tumors: expression and mutation analysis and correlation with clinical parameters. Br J Cancer 87: 635-644, 2002.

9. Simpson KJ, Dugan AS and Mercurio AM: Functional analysis of the contribution of RhoA and RhoC GTPases to invasive breast carcinoma. Cancer Res 64: 8694-8701, 2004.

10. Jiang WG, Watkins G, Lane J, Cunnick GH, Douglas-Jones A, Mokbel K and Mansel RE: Prognostic value of rho GTPases and rho guanine nucleotide dissociation inhibitors in human breast cancers. Clin Cancer Res 9: 6432-6440, 2003.

11. Kleer CG, Griffith KA, Sabel MS, Gallagher G, van Golen KL, Wu ZF and Merajver SD: RhoC-GTPase is a novel tissue biomarker associated with biologically aggressive carcinomas of the breast. Breast Cancer Res Treat 93: 101-110, 2005.

12. Forget MA, Desrosiers RR, Del M, Moumdjian R, Shedid D, Berthelet $\mathrm{F}$ and Beliveau R: The expression of rho proteins decreases with human brain tumor progression: potential tumor markers. Clin Exp Metastasis 19: 9-15, 2002.

13. Schnelzer A, Prechtel D, Knaus U, Dehne K, Gerhard M, Graeff H, Harbeck N, Schmitt M and Lengyel E: Rac1 in human breast cancer: overexpression, mutation analysis, and characterization of a new isoform, Rac1b. Oncogene 19: 3013-3020, 2000.

14. Jakobisiak M and Golab J: Potential antitumor effects of statins (Review). Int J Oncol 23: 1055-1069, 2003.

15. Fritz G: HMG-CoA reductase inhibitors (statins) as anticancer drugs (Review). Int J Oncol 27: 1401-1409, 2005

16. Mazieres J, Antonia T, Daste G, Muro-Cacho C, Berchery D, Tillement V, Pradines A, Sebti S and Favre G: Loss of RhoB expression in human lung cancer progression. Clin Cancer Res 10: 2742-2750, 2004

17. Bektic J, Pfeil K, Berger AP, Ramoner R, Pelzer A, Schafer G, Kofler K, Bartsch G and Klocker H: Small G-protein RhoE is underexpressed in prostate cancer and induces cell cycle arrest and apoptosis. Prostate 64: 332-340, 2005.

18. Liu A, Cerniglia GJ, Bernhard EJ and Prendergast GC: RhoB is required to mediate apoptosis in neoplastically transformed cells after DNA damage. Proc Natl Acad Sci USA 98: 6192-6197, 2001.

19. Fritz G and Kaina B: Ras-related GTPase RhoB forces alkylationinduced apoptotic cell death. Biochem Biophys Res Commun 268: 784-789, 2000.

20. Schmitz AA, Govek EE, Bottner B and Van Aelst L: Rho GTPases: signaling, migration, and invasion. Exp Cell Res 261: $1-12,2000$.

21. Overbeck AF, Brtva TR, Cox AD, Graham SM, Huff SY, Khosravi-Far R, Quilliam LA, Solski PA and Der CJ: Guanine nucleotide exchange factors: activators of Ras superfamily proteins. Mol Reprod Dev 42: 468-476, 1995.

22. Minard ME, Ellis LM and Gallick GE: Tiam1 regulates cell adhesion, migration and apoptosis in colon tumor cells. Clin Exp Metastasis 23: 301-313, 2006.

23. Chumbalkar VC, Subhashini C, Dhople VM, Sundaram CS, Jagannadham MV, Kumar KN, Srinivas PN, Mythili R, Rao MK, Kulkarni MJ, et al: Differential protein expression in human gliomas and molecular insights. Proteomics 5: 1167-1177, 2005.

24. Buchanan FG, Elliot CM, Gibbs M and Exton JH: Translocation of the Rac1 guanine nucleotide exchange factor Tiam1 induced by platelet-derived growth factor and lysophosphatidic acid. J Biol Chem 275: 9742-9748, 2000.

25. Leeuwen FN, Kain HE, Kammen RA, Michiels F, Kranenburg OW and Collard JG: The guanine nucleotide exchange factor Tiam1 affects neuronal morphology; opposing roles for the small GTPases Rac and Rho. J Cell Biol 139: 797-807, 1997. 
26. Sander EE, ten Klooster JP, van Delft S, van der Kammen RA and Collard JG: Rac downregulates Rho activity: reciprocal balance between both GTPases determines cellular morphology and migratory behavior. J Cell Biol 147: 1009-1022, 1999.

27. Hordijk PL, ten Klooster JP, van der Kammen RA, Michiels F, Oomen LC and Collard JG: Inhibition of invasion of epithelial cells by Tiam1-Rac signaling. Science 278: 1464-1466, 1997.

28. Sander EE, van Delft S, ten Klooster JP, Reid T, van der Kammen RA, Michiels F and Collard JG: Matrix-dependent Tiam1/Rac signaling in epithelial cells promotes either cell-cell adhesion or cell migration and is regulated by phosphatidy-linositol 3kinase. J Cell Biol 143: 1385-1398, 1998.

29. Engers R, Mueller M, Walter A, Collard JG, Willers R and Gabbert HE: Prognostic relevance of Tiam1 protein expression in prostate carcinomas. Br J Cancer 95: 1081-1086, 2006.
30. Michiels F, Stam JC, Hordijk PL, van der Kammen RA, Ruuls-Van Stalle L, Feltkamp CA and Collard JG: Regulated membrane localization of Tiam1, mediated by the NH2-terminal pleckstrin homology domain, is required for Rac-dependent membrane ruffling and C-Jun NH2-terminal kinase activation. J Cell Biol 137: 387-398, 1997.

31. Brozovic A and Osmak M: Activation of mitogen-activated protein kinases by cisplatin and their role in cisplatin-resistance. Cancer Lett 251: 1-16, 2007.

32. Hayakawa J, Depatie C, Ohmichi M and Mercola D: The activation of c-Jun NH2-terminal kinase (JNK) by DNAdamaging agents serves to promote drug resistance via activating transcription factor 2 (ATF2)-dependent enhanced DNA repair. J Biol Chem 278: 20582-20592, 2003. 